\title{
The Turkish League Against Rheumatism Recommendations for the Management of Hand Osteoarthritis Under Guidance of the Current Literature and 2018 European League Against Rheumatism Recommendations
}

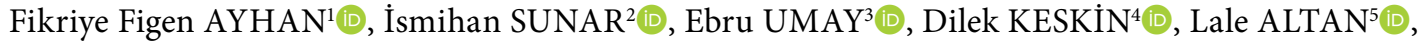

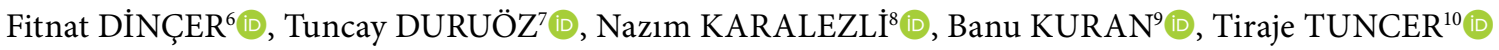 \\ ${ }^{1}$ Department of Physical Medicine and Rehabilitation, Uşak University, School of Medicine, Uşak, Turkey \\ ${ }^{2}$ Department of Physical Medicine and Rehabilitation, Division of Rheumatology, Ankara University, School of Medicine, Ankara, Turkey \\ ${ }^{3}$ Department of Physical Therapy and Rehabilitation, Health Sciences University, \\ Dışkapı Yıldırım Beyazıt Training and Research Hospital, Ankara, Turkey \\ ${ }^{4}$ Department of Physical Medicine and Rehabilitation, Kırıkkale University, School of Medicine, Kirıkkale, Turkey \\ ${ }^{5}$ Department of Physical Medicine and Rehabilitation, Division of Rheumatology, Uludă University, School of Medicine, Bursa, Turkey \\ ${ }^{6}$ Department of Physical Medicine and Rehabilitation, Hacettepe University, School of Medicine, Ankara, Turkey \\ ${ }^{7}$ Department of Physical Medicine and Rehabilitation, Division of Rheumatology, Marmara University, School of Medicine, Istanbul, Turkey \\ ${ }^{8}$ Department of Orthopedics, Division of Hand Surgery, Muğla Sitkı Koçman University, School of Medicine, Muğla, Turkey \\ ${ }^{9}$ Department of Physical Medicine and Rehabilitation, Health Sciences University, Şişli Hamidiye Etfal Training and \\ Research Hospital, İstanbul, Turkey \\ ${ }^{10}$ Department of Physical Medicine and Rehabilitation, Division of Rheumatology, Akdeniz University, School of Medicine, Antalya, Turkey
}

\begin{abstract}
Objectives: This study aims to explore the accordance to the 2018 European League Against Rheumatism (EULAR) recommendations for the management of hand osteoarthritis (OA) among the Turkish League Against Rheumatism (TLAR) expert panel and composition of TLAR recommendations for the management of hand OA under guidance of the current literature.

Materials and methods: The TLAR convener designated an expert panel of 10 physicians experienced in hand OA for this process. The 2018 EULAR recommendations for the management of hand $\mathrm{OA}$ and the systematic review of the literature were sent to the expert panel via e-mails. The e-mail process which included Delphi round surveys was completed. The EULAR standard operational procedure Appraisal of Guidelines for Research and Evaluation II was followed. The level of agreement was calculated for each item and presented as mean, standard deviations, minimum and maximum and comparisons of 2018 EULAR recommendations were performed.

Results: Five overarching principles and 10 recommendations were discussed. Revisions were held for the sixth, seventh, and ninth recommendations with lowest level of agreements. These recommendations were revised in accordance with suggestions from the experts and re-voted. The revised forms were approved despite the lack of statistically significant difference between these forms $(p=0.400, p=0.451, p=0.496$, respectively). Except for the ninth recommendation about surgery $(p=0.008)$, no significant difference in level of agreements was observed between the EULAR and TLAR hand $\mathrm{OA}$ recommendations. The $11^{\text {th }}$ recommendation about paraffin bath was added.

Conclusion: The optimal treatment of hand OA consists of personalized non-pharmacological (self-management, exercise, splint), pharmacological (topical non-steroidal anti-inflammatory drugs as the first choice), and interventional procedures (only for refractory cases) based on shared decision between the patient and physician. TLAR hand OA recommendations were created mainly based on the most recent literature and the last EULAR hand OA management recommendations, which are widely approved among the TLAR experts.

Keywords: Guideline, hand, management, osteoarthritis, treatment.
\end{abstract}

Received: August 29, 2019 Accepted: September 14, 2019 Published online: January 08, 2020

Correspondence: Fikriye Figen Ayhan, MD. Uşak Üniversitesi Tıp Fakültesi Fiziksel Tıp ve Rehabilitasyon Anabilim Dalı, 1 Eylül Kampüsü, 64200 Uşak, Türkiye. Tel: +90 276- $2212121 / 3000$ e-mail: figen.ayhan@usak.edu.tr

\section{Citation:}

Ayhan FF, Sunar I, Umay E, Keskin D, Altan L, Dinçer F, et al. The Turkish League Against Rheumatism Recommendations for the Management of Hand Osteoarthritis Under Guidance of the Current Literature and 2018 European League Against Rheumatism Recommendations. Arch Rheumatol 2020;35(3):309-320. 
Hand osteoarthritis (OA) is the most common form of osteoarthritis with high age-standardized prevalence compared to other joints such as knee and hip osteoarthritis. ${ }^{1}$ The prevalence is the highest among older females and it is predicted that the prevalence will increase with aging. ${ }^{1-3}$ Hand $\mathrm{OA}$ causes poor quality of life (QoL) with functional disability, activity limitation and participation restriction in daily and social life by a wide range of symptoms and findings such as pain, morning stiffness, tenderness and swelling. ${ }^{4}$ Studies reported that direct costs related to hand OA constitute a serious socioeconomic burden., ${ }^{4,5}$ Due to the fact that this disease is seen in advanced age, the indirect cost reasons such as additional comorbidities have increased the interest in its management. ${ }^{6}$ Thus, the first guideline of recommendations for the management of hand OA was published in 2007 by the European League Against Rheumatism (EULAR). ${ }^{7}$ Then, an expert group consisting of occupational therapists developed a specific management guide based on non-pharmacological treatment management in 2011, and this guide indicated the lack of high-quality studies. ${ }^{8}$ Following this guideline, several groups including the American College of Rheumatology (2012), Italian Society for Rheumatology (2013) and National Institute for Health and Care Excellence group (2014) created guidelines of recommendations for the management of knee, hip and hand OA, based on the recommendations of EULAR as well as the new treatment approaches and methods. ${ }^{9-11}$ Although each group included new studies, they produced guidelines with similar recommendations while almost all groups indicated the need for high level of evidence. Unfortunately, the majority of the recommendations consisted of evidence of expert opinion.

It is well known that the science of medicine goes even further and takes us one step forward every day. Approximately 400 studies have been carried out per year over the last four years after the publication of the last guideline. The EULAR felt the need to update its recommendations in $2007,{ }^{7}$ particularly due to the availability of pharmacological studies involving many new molecules and new treatment options. Therefore, the 2018 EULAR hand OA update management recommendation guideline has been presented with the latest studies in the literature. ${ }^{12}$ After this guideline, many studies have been published about the treatment of hand $\mathrm{OA}$, including combined non-pharmacological interventions such as education, exercise, splinting, and paraffin bath therapy, ${ }^{13-15}$ intra-articular injections, ${ }^{16}$ biologic disease modifying anti-rheumatic drugs (DMARDs), ${ }^{17-19}$ and surgery. ${ }^{20}$ Furthermore, two systematic reviews about splinting and surgery in thumb base OA have also been published recently. ${ }^{21,22}$

Management recommendation guidelines are systematically developed statements to assist clinicians in appropriate healthcare decisions for specific clinical conditions. ${ }^{23}$ Each country has genetic, socioeconomic and cultural differences. Therefore, it is necessary for physicians to investigate any update and compliance with these recommendations in order to achieve the "most reliable way" by putting forward the applicability in the guidance of the national scientific organization.

In this study, we aimed to explore the accordance to the 2018 EULAR recommendations $^{12}$ for the management of hand OA among the Turkish League Against Rheumatism (TLAR) expert panel and composition of TLAR recommendations for the management of hand OA under guidance of the current literature.

\section{MATERIALS AND METHODS}

This project was conducted by The Turkish League Against Rheumatism (TLAR) Hand OA expert panel between December 2018 and March 2019. The TLAR assigned a task committee comprising of ten experts throughout Turkey who take care of patients with hand OA intensively in daily practice. The expert panel comprised of four academic staff working in the rheumatology division of physical and rehabilitation medicine (PRM) department, four academic staff experienced in hand rehabilitation working in PRM department, one pain medicine specialist, and one hand surgeon. The corresponding author of the 2018 EULAR recommendations for the management of hand $\mathrm{OA}^{12}$ was informed by e-mail for this process 
by the first author. Systematic literature review informing the 2018 update of the EULAR recommendations for the management of hand $\mathrm{OA}^{24}$ was also suggested to be used by the corresponding author. We followed the EULAR standard operational procedure Appraisal of Guidelines for Research and Evaluation II. ${ }^{25}$

We included studies published after the publication of the 2018 EULAR recommendations for the management of hand $\mathrm{OA}$ and prior to the beginning of the Delphi rounds for the current process. The terms 'hand', 'osteoarthritis' 'management', and 'treatment' were used to reach the most recent data in PubMed, Cochrane, MEDLINE, and Scopus between June 2018 and January 2019 by two-independent researchers. Inclusion criteria were studies with high level of evidence (meta-analyses, systematic reviews, randomized controlled trials, controlled clinical trials published in journals indexed in the Science Citation Index or Science Citation Index Expanded) and a minimum follow-up time of two months. The fourth author translated the 2018 update of the EULAR recommendations for the management of hand OA. ${ }^{12}$

The Delphi rounds performed via e-mails were completed between 06 January 2019 and 12 February 2019. Some open-ended questions regarding panel attendees' attitude and approach towards patients or drugs in follow-up of patients with hand OA were sent to the expert panel via e-mails by the first author in Delphi-1 round. The original articles and the Turkish translations of abstracts were also sent to experts at the same time. The original overarching principles and recommendations of 2018 EULAR hand OA guideline and fulltext Turkish translation were sent to the panel experts in Delphi-2 round. All principles and recommendations were voted by experts with numbers of 0-10, 0 reflecting 'I totally disagree', and 10 reflecting 'I totally agree'. A 75\% majority was required to approve the statement. In case of disagreement, discussion was resumed and changes to the statement were made. The second voting round required a $67 \%$ majority, and if the formulation remained disagreed, an additional round of discussion followed. The third voting round required only 50\% support for approval of the statement. The wording of the statements was considered final after the $e$-mail conversations.

\section{Statistical analysis}

Descriptive statistics of SPSS version 11.5 (SPSS Inc., Chicago, IL, USA) software was used for statistical analyses by the biostatistician. The level of agreements were calculated for each item and presented as mean, standard deviation, and minimum-maximum. The level of agreement of revised recommendations was compared to the level of agreement of original recommendations (first and second TLAR votings) using the paired t-test due to inhomogeneity of variances. The student t-test was also used for comparison of each item's level of agreements of the 2019 TLAR recommendations and the 2018 EULAR recommendations for the management of hand $\mathrm{OA}$. $\mathrm{P}$ values less than 0.05 were considered as statistically significant.

\section{RESULTS}

The 2019 TLAR recommendations for the management of hand OA were composed which were mainly based on 2018 EULAR management recommendations for hand OA (Table 1). We also took into consideration high-evidenced novel studies published thereafter.

\section{Overarching principles}

Five overarching principles were discussed and accepted by the expert panel. The highest level of agreement was obtained in overarching principles $\mathrm{A}$ and $\mathrm{C}$.

Principle A: The main goal in treatment of hand $O A$ is controlling the symptoms including pain and stiffness, optimizing the hand function, and maximizing the activity participation and quality of life.

The patients with hand OA have difficulty in daily living activities because of pain, stiffness, decreased mobility and significant loss in hand grip strength. ${ }^{26,27}$ The main complaints of patients with hand $\mathrm{OA}$ are pain, loss of dexterity in some fine motor skills, and some cosmetic concerns about changing finger shapes. Primary priority is to achieve independence in self-care and activities of daily living. ${ }^{12}$ Symptomatic hand OA may be similar to rheumatoid hands regarding pain and health related QoL. ${ }^{28}$ It has been 
reported that the limitations in daily activities related to hand $\mathrm{OA}$ are major determinants of reduced health-related QoL. ${ }^{29}$

Principle B: Every patient should be provided with information regarding the disease and its course, self-management principles and therapeutic options.

We should give some information to patients with hand $\mathrm{OA}$ about the nature and course of the disease as well as non-pharmacological, pharmacological, and surgical treatment choices. ${ }^{30}$ It has been reported that self-management approaches are also useful for managing pain in these patients. ${ }^{31}$

Principle $\boldsymbol{C}$ : The treatment of hand $O A$ should be personalized considering the localization and severity of $O A$ and comorbid diseases.

Thumb base or first carpometacarpal (first $\mathrm{CMC}$ ) joint $\mathrm{OA}$ is more painful and it leads to decrease of hand grip strength although distal interphalangeal (DIP) joints are more commonly affected. ${ }^{32,33}$ Comorbidities should be considered because musculoskeletal (e.g., connective tissue disease) and non-musculoskeletal (e.g., pulmonary and cardiovascular disease) comorbidities are associated with disease burden. ${ }^{6}$ Since the distribution and severity of involvement pattern are affected by sex, job, and comorbidities, the management of hand $\mathrm{OA}$ should be individualized. ${ }^{1,12}$

Principle D: The treatment of hand $O A$ should rely on a shared decision between the patient and health professionals.

The concept of shared-decision between patient and health professionals is a healthcare approach about sharing information to reach a consensus on treatment decision. The treatment choices should be shared with patients considering individual needs ${ }^{11,12,24}$ because this approach is both effective ${ }^{34}$ and cost-effective. ${ }^{35}$ In our open ended Delphi questions, 90\% of panelists reported taking shared decisions with the patients while only one expert stated making 'partially' shared therapeutic decisions. They emphasized that several chronic rheumatic diseases which require high patient compliance to attain treatment success should contain co-decisions between the patient and the health professionals.
Principle E: Optimal treatment of hand $O A$ generally entails a multi-disciplinary approach. Pharmacological options and surgery should be considered in addition to non-pharmacological treatment.

Hand OA is a chronic, heterogeneous, painful and sometimes disabling disease. In our country, specialties of PRM, rheumatology (both internal medicine and PRM), pain medicine, and hand surgery (orthopedics/plastic surgery) are mainly interested in hand OA. Non-pharmacological options such as education, exercises, splinting, and paraffin, ${ }^{13-15,21}$ or pharmacological interventions such as topical and systemic non-steroidal anti-inflammatory drugs (NSAIDs), local injections, and surgical procedures ${ }^{11,12,36}$ should be considered in the management of hand OA.

\section{Recommendations}

Ten recommendations were discussed and one recommendation was added; totally, 11 recommendations were listed in Table 1 . The sixth, seventh, and ninth recommendations attained the lowest level of agreement and demands for revision were requested from the expert panel. These recommendations were revised by the expert panel and re-voted. Although there were no statistically significant differences between the revised and original forms, the revised forms were recognized ( $p=0.400, p=0.451, p=0.496$, respectively). The $11^{\text {th }}$ recommendation was integrated to the present recommendations because of two randomized controlled trials. ${ }^{14,37}$ The comparisons of level of agreements for the TLAR and EULAR hand OA treatment recommendations were shown in Table 2.

Except for the ninth recommendation about surgery, no significant difference was observed between level of agreements of the TLAR and EULAR recommendations for the management of hand OA. The seventh item of EULAR hand $\mathrm{OA}$ recommendation about intra-articular treatment has been totally changed and new $11^{\text {th }}$ recommendation about paraffin bath has been added by TLAR experts.

Recommendation 1: Every patient should be educated and trained in terms of ergonomic principles, activity breaks, and use of assistive devices. 
Table 1. Turkish League Against Rheumatism recommendations for management of hand osteoarthritis

Overarching Principles
A The main goal in treatment of hand OA is controlling the symptoms including pain and stiffness, optimizing the hand function,
and maximizing the activity participation and quality of life.
B therapeutic options.
C The treatment of hand OA should be personalized considering the localization and severity of OA and comorbid diseases.
D The treatment of hand OA should rely on a shared decision between the patient and health professionals.
E $\begin{aligned} & \text { Optimal treatment of hand OA generally entails a multi-disciplinary approach. Pharmacological options and surgery should be } \\ & \text { considered in additional to non-pharmacological treatment. }\end{aligned}$
considered in additional to non-pharmacological treatment.

\section{Recommendations}

1 Every patient should be educated and trained in terms of ergonomic principles, activity breaks, and use of assistive devices.

2 Exercise should be considered for all patients to enhance function and muscle strength in addition to reducing pain.

3 Patients with thumb base OA should be considered for use of splints in order to alleviate symptoms. Long duration of use is encouraged.

4 Topical treatment is preferred over systematic treatment due to safety concerns. Topical NSAIDs are the first choice of treatment

5 Oral analgesics, particularly NSAIDs, should be used for a short term for alleviation of symptoms.

6 Revised 6: In patients who do not attain adequate benefit from paracetamol or topical/systemic NSAIDs or whom these agents are contraindicated, chondroitin sulfate may be used to reduce pain and improve functions. Original 6: Chondroitin sulfate may be used in patients with hand OA for pain relief and improvement in functioning.

$7 \quad$ Changed 7: In patients with first CMC OA and/or IP OA who do not attain adequate benefit from paracetamol or topical/systemic NSAIDs or whom these agents are contraindicated, intra-articular treatments may be considered.

Original 7: Intra-articular injections of glucocorticoids should not generally be used in patients with hand OA, but may be considered in patients with painful interphalangeal joints.

8 Conventional synthetic or biologic DMARDs should not be used in patients with hand OA

9 Revised 9: Surgical interventions may be considered in patients with structural abnormalities when other treatment modalities are inadequate to alleviate the pain. Arthrodesis (for DIP joints), arthrodesis or arthroplasty (for PIP joints), arthroplasty (for MCP joints), trapeziectomy+LRTI, arthroplasty or arthroscopic partial trapeziectomy (for first CMC joint) may be considered. Rehabilitation programs following first CMC arthroplasty are efficient and safe in pain relief and improvement of function and grip strength.

Original 9: Surgery should be considered for patients with structural abnormalities when other treatment modalities have not been sufficiently effective in relieving pain. Trapeziectomy should be considered in patients with thumb base OA and arthrodesis or arthroplasty in patients with IP OA.

10 The long-term follow-up of patients with hand OA should be adjusted according to patients' individual requirements.

11 New: Paraffin may be considered as a safe and efficient non-pharmacological treatment modality relieving the pain and improving grip strength in the short term.

OA: Osteoarthritis; NSAIDs: Non-steroidal anti-inflammatory drugs; first CMC: First carpometacarpal; IP: Interphalangeal; DMARDs: Disease modifying anti-rheumatic drugs; DIP: Distal interphalangeal; PIP: Proximal interphalangeal; MCP: Metacarpophalangeal; LRTI: Ligament reconstruction and tendon interposition.

Eighty percent of panel voters suggested patient education about ergonomics, short breaks, or thumb spica splint for thumb base OA, while 30\% also recommended the use of transcutaneous electrical nerve stimulation and local heat. Education is an effective and safe nonpharmacological intervention, particularly when combined with other modalities such as splinting and exercises. ${ }^{13-15}$
Recommendation 2: Exercise should be considered for all patients to enhance function and muscle strength in addition to reducing pain.

Although $100 \%$ of the experts stated that hand exercises are important and improve function and muscle strength, $80 \%$ of them reported prescribing exercise programs to all patients with hand OA. Seventy percent believed that 
Table 2. Comparison of level of agreements for Turkish League Against Rheumatism and European League Against Rheumatism hand osteoarthritis treatment recommendations

\begin{tabular}{|c|c|c|c|c|c|c|c|}
\hline \multirow[b]{2}{*}{ Overarching principles } & \multirow[b]{2}{*}{$\mathrm{LoE}^{*}$} & \multicolumn{2}{|c|}{ TLAR LoA } & \multicolumn{2}{|c|}{ TLAR LoA for the revised items } & \multirow{2}{*}{$\frac{\text { EULAR LoA }}{\text { Mean } \pm \text { SD }}$} & \multirow[b]{2}{*}{$p$} \\
\hline & & Mean \pm SD & Min-Max & Mean \pm SD & Min-Max & & \\
\hline A & & $9.90 \pm 0.32$ & $9-10$ & & & $9.7 \pm 0.7$ & 0.300 \\
\hline B & & $9.70 \pm 0.67$ & $8-10$ & & & $9.8 \pm 0.8$ & 0.725 \\
\hline C & & $9.90 \pm 0.32$ & $9-10$ & & & $9.9 \pm 0.2$ & 1.00 \\
\hline $\mathrm{D}$ & & $9.10 \pm 0.99$ & $7-10$ & & & $9.6 \pm 1.1$ & 0.226 \\
\hline E & & $9.00 \pm 1.25$ & $7-10$ & & & $9.3 \pm 1.2$ & 0.538 \\
\hline \multicolumn{8}{|l|}{ Recommendations } \\
\hline 1 & $1 b$ & $9.40 \pm 1.07$ & $7-10$ & & & $9.3 \pm 1.1$ & 0.815 \\
\hline 2 & 1a & $9.50 \pm 0.97$ & $7-10$ & & & $9.1 \pm 1.6$ & 0.411 \\
\hline 3 & $1 b$ & $8.90 \pm 1.29$ & $7-10$ & & & $9.3 \pm 1.0$ & 0.399 \\
\hline 4 & $1 b$ & $8.60 \pm 1.65$ & $5-10$ & & & $8.6 \pm 1.8$ & 1.00 \\
\hline 5 & $1 \mathrm{a}$ & $9.65 \pm 0.67$ & $8-10$ & & & $9.4 \pm 0.9$ & 0.405 \\
\hline 6 & $1 b$ & $6.55 \pm 1.86$ & $3-10$ & $7.45 \pm 2.45$ & $1.5-10^{* * * *}$ & $7.3 \pm 2.7$ & 0.388 \\
\hline 7 & $1 b$ & $6.70 \pm 2.2$ & $2-10$ & $7.60 \pm 2.18$ & $2.5-10^{* * * *}$ & $7.9 \pm 2.4$ & 0.189 \\
\hline 8 & $1 \mathrm{a}$ & $8.60 \pm 2.32$ & $3-10$ & & & $8.8 \pm 1.8$ & 0.814 \\
\hline 9 & $5,1 a$ & $7.60 \pm 1.71$ & $5-10$ & $6.85 \pm 2.58$ & $1.5-10 * * *$ & $9.4 \pm 1.4 * *$ & 0.008 \\
\hline 10 & 5 & $9.80 \pm 0.63$ & $8-10$ & & & $9.5 \pm 1.7$ & 0.500 \\
\hline New 11 & $1 \mathrm{a}, 1 \mathrm{~b}$ & $8.90 \pm 0.99$ & $7-10$ & & & & \\
\hline
\end{tabular}

TLAR: Turkish League Against Rheumatism; LoA: Level of agreement; EULAR: European League Against Rheumatism; * LoE: Level of evidence; SD: Standard deviation; Min: Minimum; Max: Maximum; 1a: Systematic review of randomized controlled trials; $1 \mathrm{~b}$ : Randomized controlled trial; 2a: Systematic review of cohort studies; $2 \mathrm{~b}$ : Cohort study; 3a: Systematic review of case control studies; $3 \mathrm{~b}$ : Case control study; 4 : Case series; 5 : Expert opinion; ${ }^{* *}$ p $<0.05$ statistical significance; ${ }^{* * * *} \mathrm{p}>0.05$ statistical insignificance.

hand exercises are effective in pain relief. The type of prescribed exercises may be listed as range of motion (90\%), strengthening (80\%), stretching (60\%), and stabilization (20\%) exercises. Exercise programs are different for the first CMC joint from interphalangeal (IP) joint OA. Hand exercises are effective with small effect size, ${ }^{38}$ and it has been reported that they are more effective when combined with other non-pharmacological treatment modalities such as education, splinting, ergonomics, or paraffin bath. ${ }^{13-15}$

Recommendation 3: Patients with thumb base OA should be considered for use of splints in order to alleviate symptoms. Long duration of use is encouraged.

Ninety percent of experts regularly prescribed while $10 \%$ sometimes prescribed thumb spica splint (or short opponens splint) in patients with first CMC OA. Fifty percent suggested short-term splint while the remaining 50\% suggested longterm splint. Thumb splints are effective on pain and function in medium-term (3-12 months) with low level of evidence 21 and generally combined with non-pharmacological treatments. ${ }^{15}$

Recommendation 4: Topical treatment is preferred over systematic treatment due to safety concerns. Topical NSAIDs are the first choice of treatment.

While $90 \%$ of experts preferred topical treatments because of safety concerns, particularly in older patients with many comorbidities and poly-pharmacies, $10 \%$ preferred combined topical NSAIDs and paracetamol prescription. Topical NSAIDs are effective to reduce pain and improve function in osteoarthritis. 7,8,11,12,39 Topical capsaicin was not accepted as a favored analgesic because of local adverse effects (disturbing burning and stinging sensation).

Recommendation 5: Oral analgesics, particularly NSAIDs, should be used for a short term for alleviation of symptoms.

One hundred percent of experts regularly prescribed oral analgesics to patients 
with hand OA. While 60\% of experts prescribed paracetamol or NSAIDs, $40 \%$ prescribed only NSAIDs for a short term. Oral NSAID is effective but not safe due to gastrointestinal, renal, and cardiovascular adverse effects, particularly in elderly patients with OA. ${ }^{7,8,11,12}$ TLAR experts suggested oral NSAIDs in limited duration (preferably on-demand). A weak analgesic, paracetamol may be used in contraindicated conditions of oral NSAIDs such as renal disease.

Revised Recommendation 6: In patients who do not attain adequate benefit from paracetamol or topical/systemic NSAIDs or whom these agents are contraindicated, chondroitin sulfate may be used to reduce pain and improve functions.

Seventy-five percent of experts advocated the effects of both chondroitin sulfate and (only chondroitin 75\%) or glucosamine sulfate (only glucosamine 40\%) for relieving pain and increasing function. However, these over-the-counter nutraceutical products are expensive, not covered by social security systems and not superior to other pharmacological interventions of OA. There was only one study about the structural effects of chondroitin polysulfate (not sulfate) in patients with hand $\mathrm{OA}^{40}$ and acetaminophen consumption was not different between groups in another study. ${ }^{41}$ Therefore, we considered to change this recommendation as "chondroitin sulfate may be prescribed to the patients who do not attain adequate benefit from acetaminophen (paracetamol) or topical/systemic NSAIDs or whom these agents are contraindicated". Although there was no statistical difference between the level of agreements of original and revised items, the revised item was accepted.

Changed Recommendation 7: In patients with first CMC OA and/or IP OA who do not attain adequate benefit from paracetamol or topical/systemic NSAIDs or whom these agents are contraindicated, intra-articular treatments may be considered.

While $75 \%$ of experts believed in the benefit of first CMC glucocorticoid injection in painful thumb base OA resistant to other treatments, the remaining $25 \%$ did not believe in the beneficial effect of intra-articular injection. However, if there is just one inflamed joint, local injection can be a good option. The hand surgeon suggested that since IP joints are smaller than first CMC joint, sometimes the injection material goes to the periarticular area in the narrowed joint space without imaging modalities. ${ }^{16}$ It was reported in many randomized controlled trials that intra-articular interventions including platelet-rich plasma compared to glucocorticoid ${ }^{16}$ or glucocorticoid compared to hyaluronic $\mathrm{acid}^{42}$ or hyaluronic acid compared to saline ${ }^{43}$ injections were more effective in first CMC OA. Although there was no statistical difference between the level of agreements of original and revised items, the revised item was accepted.

Recommendation 8: Conventional synthetic or biologic DMARDs should not be used in patients with hand $O A$.

Seventy-five percent of experts had some questionable experiences including conventional synthetic or biologic DMARDs or glucocorticoids in hand OA. There was no evidence about these pharmacological interventions in hand OA. ${ }^{12}$ However, while $40 \%$ of experts prescribed csDMARDs and colchicine, $30 \%$ of them used hydroxychloroquine (HCQ) and remaining 10\% used colchicine. There are some clinical trials yielding various results on the effects of $\mathrm{HCQ}$, methotrexate and apremilast in hand and knee OA.44-46 Although HCQ was not found to be more effective than placebo for pain relief in patients with radiographic hand $\mathrm{OA}$ in a randomized, double-blind, placebo-controlled clinical trial, this failure was stated to have been a result of the dose restrictions. The authors also propound that missing the window of opportunity for HCQ to have therapeutic benefit by enrolling relatively late stage patients may be another obstacle since early OA may be more inflammatory and prone to benefit from DMARDs than established OA. ${ }^{44}$ Regardless, HCQ is used for erosive hand OA mainly based on expert opinion in daily practice.

Revised Recommendation 9: Surgical interventions may be considered in patients with structural abnormalities when other treatment modalities are inadequate to alleviate the pain. Arthrodesis (for DIP joints), arthrodesis or arthroplasty [for proximal interphalangeal (PIP) joints], arthroplasty [for metacarpophalangeal (MCP) joints], trapeziectomy+ligament reconstruction and tendon interposition (LRTI), and arthroplasty or arthroscopic partial 
trapeziectomy (for first CMC joint) may be considered. Rehabilitation programs following first CMC arthroplasty are efficient and safe in pain relief and improvement of function and grip strength.

Seventy percent of experts stated that they preferred surgery as the last option in patients with structural abnormalities when other treatment modalities are inadequate to alleviate the pain. There are many surgical techniques for first CMC OA including trapeziectomy+LRTI, arthroplasty or arthroscopic partial trapeziectomy in patients with hand OA having retractable joint pain. Arthrodesis for DIP joints, arthrodesis or arthroplasty for PIP joints, and arthroplasty for $\mathrm{MCP}$ joints are the main surgical techniques for these patients. New studies have also been published with good results about total first CMC arthroplasty, trapeziectomy, LRTI, total endoprosthesis, and resurfacing arthroplasty for thumb base OA and silicone MCP arthroplasty for MCP OA, after the publication of the systematic review of 2018 EULAR hand OA management guideline. ${ }^{20-22}$ We added the sentence about postoperative rehabilitation, taking the recently published systematic review into consideration: ${ }^{47}$ "Early active mobilization following first CMC arthroplasty are efficient and safe in pain relief and improvement of function and grip strength". Although there was no statistical difference between the level of agreement values of original and revised items, the revised item was accepted.

Recommendation 10: The long-term follow-up of patients with hand OA should be adjusted according to patients' individual requirements.

Ninety percent of experts stated that they followed-up some patients according to the patients' individual requirements. 'Individual needs' depend on existence of erosive OA, symptom severity, re-evaluation requirements and patient's wishes. Although, to our knowledge, there was no study about the effectiveness of long-term follow-up in hand $\mathrm{OA}$, some patients with erosive $\mathrm{OA}$ phenotype may have more painful, clinical and structural progressive disease. ${ }^{48}$ The follow-up may increase the adherence to non-pharmacological and pharmacological treatment in these cases.

Added Recommendation 11: Paraffin may be considered as a safe and efficient non- pharmacological treatment modality relieving the pain and improving grip strength in the short-term.

High quality evidence shows that unimodal and multimodal physical therapy treatments can result in clinically worthwhile improvements in pain and function for patients with base of thumb OA. ${ }^{49}$ It has been reported that paraffin is an effective modality of physical therapy, particularly when combined with exercise. ${ }^{14,37}$ This recommendation was dropped in 2018 EULAR hand OA guideline; however, we should add it because of the clear evidence from the two mentioned randomized controlled trials.

\section{DISCUSSION}

The present article presents the first TLAR hand OA management recommendations including five overarching principles and 11 recommendations. We have added one recommendation, two revisions and one change to the 2018 EULAR hand OA management recommendations according to the comments of TLAR expert panel and the evidences of the current literature review after 2017.

Five overarching principles of EULAR hand OA management recommendations were accepted by all experts with high level of compliance while the items representing the highest compliance are principle $\mathrm{A}$ and principle $\mathrm{C}$. As a matter of fact, our main goal for the treatment of hand $\mathrm{OA}$ is pain relief, subsequently improving hand function as much as possible, enabling maximum participation in daily living activities, and maximizing QoL (overarching principle A). The treatment of hand OA should be personalized considering the localization and severity of $\mathrm{OA}$ and comorbid diseases since thumb base and IP joint OA have different characteristics and elderly populations may have some comorbid diseases (overarching principle C). ${ }^{26-29}$ While thumb base OA may lead to more difficulties in activities of daily living, IP joint OA generally presents prominent swelling, finger deformities and subsequently cosmetic concerns.

Seven out of 10 recommendations of EULAR hand OA management guideline were accepted without revision. However, recommendation 
number six was about chondroitin sulfate and our experts have discussed this item due to unknown cost-effectivity and limited data about hand OA..$^{40,41}$ The expert panel have concluded that paracetamol, or topical and systemic NSAIDs, can be better choices compared to chondroitin sulfate for these patients. Therefore, we have added some words to the sentence "In patients who do not attain adequate benefit from paracetamol or topical/systemic NSAIDs or whom these agents are contraindicated, chondroitin sulfate may be used to reduce pain and improve functions".

The pain medicine specialist from the expert panel suggested performing a revision in the recommendation number seven. Although first $\mathrm{CMC}$ and IP joints have very small size of joint space, it was reported that intra-articular injections of these joints with or without imaging guidance have some benefit in patients with hand $\mathrm{OA}$ in clinical practice. To the best of our knowledge, there were some controlled studies favoring intra-articular treatments such as glucocorticoid, hyaluronic acid and plateletrich plasma injections for at least three-months of follow-up in patients with hand OA.16,42,43 Therefore, recommendation number seven was completely changed as: "In patients with first CMC OA and/or IP OA who do not attain adequate benefit from paracetamol or topical/ systemic NSAIDs or whom these agents are contraindicated, intra-articular treatments may be considered" as interventional pain treatments.

The hand surgeon from the expert panel suggested clear explanation for the ninth recommendation as follows: "Surgical interventions may be considered in patients with structural abnormalities when other treatment modalities are inadequate to alleviate the pain. Arthrodesis (for DIP joints), arthrodesis or arthroplasty (for PIP joints), arthroplasty (for MCP joints), trapeziectomy+LRTI, arthroplasty or arthroscopic partial trapeziectomy (for first CMC joint) may be considered". We would like to explain the type of surgical methods because of the importance of stability (mainly for first CMC and DIP joints) and need for mobility (mainly for MCP and PIP joints) concerns in different joints reported recently ${ }^{20-22}$ and to emphasize the role of postoperative rehabilitation. ${ }^{48}$ Thus, we added the sentence about hand rehabilitation as follows: "Rehabilitation programs following first $\mathrm{CMC}$ arthroplasty are efficient and safe in pain relief and improvement of function and grip strength".

The TLAR expert panel has consisted of physicians familiar to physical therapy modalities such as paraffin bath and electro-therapy. The recommendation about paraffin bath was dropped in 2018 EULAR hand OA guideline; however, we have considered to add it because of the clear evidence from two randomized controlled trials. ${ }^{14,37}$ And recently, a systematic review and meta-analysis have reported high quality evidence of unimodal and multimodal physical therapy treatments resulting in clinically worthwhile improvements in pain and function for patients with base of thumb OA. ${ }^{49}$

The EULAR has aimed to disseminate 2018 hand OA recommendations across national societies for healthcare providers. ${ }^{12,24}$ Also, for this aim, several national societies worldwide have exhibited different point of views. ${ }^{9-11}$ Certain societies including the Pan-American League of Associations for Rheumatology (PANLAR) have published their consensus report on hand, hip and knee OA. ${ }^{50}$ Therefore, we also made an effort to perform compliance and adaptations of the 2018 EULAR hand OA recommendations to develop national recommendations by the TLAR.

Similar to our recommendations, 2016 PANLAR hand OA recommendations have also consisted of non-pharmacological (education, exercises and splint), pharmacological (topical NSAIDs, paracetamol, oral NSAIDs, glucosamine and chondroitin sulfate, intra-articular steroids or hyaluronic acid for symptomatic first CMC joint), and surgical interventions (LRTI, and trapeziectomy, arthroplasty, and arthrodesis in severe first CMC OA after conservative treatments have failed).

The strength of these recommendations was the panel members consisting of 10 physicians intensively interested in hand pain, arthritis, rehabilitation, and surgery in clinical practices and universities in our country. Totally, eight physicians were academic staff in rheumatology \& physical and rehabilitation medicine departments, equally. The remaining two physicians were also academicians working intensively in hand surgery and pain medicine divisions familiar to difficult cases of arthritic pain needing interventional procedures and surgery. One limitation of the 
current study is the lack of quality in some studies we referred to (recommendation number six and recommendation number eight).

In conclusion, we have performed some revisions, additions, and changes in the light of the current literature and the TLAR expert perspective. We have recommended chondroitin sulfate and/or intra-articular treatments only in patients who do not attain adequate benefit from paracetamol or topical/systemic NSAIDs or whom these agents are contraindicated. We have also added both paraffin bath and postoperative rehabilitation as recommendations in the setting of physical therapy and hand rehabilitation. If all of these non-surgical treatments have failed, surgery may be the last option for patients with severe hand OA. Since the aging and handworking world population with hand OA may need new and effective treatments in the future, our society plans to update our recommendations periodically.

\section{Acknowledgements}

We would like to thank the biostatistician, Atilla Halil Elhan, MD, Professor, for his thoughtful comments and efforts towards improving our statistical analysis of data.

\section{Declaration of conflicting interests}

The authors declared no conflicts of interest with respect to the authorship and/or publication of this article.

\section{Funding}

The authors received no financial support for the research and/or authorship of this article.

\section{REFERENCES}

1. Haugen IK, Englund M, Aliabadi P, Niu J, Clancy M, Kvien TK, et al. Prevalence, incidence and progression of hand osteoarthritis in the general population: the Framingham Osteoarthritis Study. Ann Rheum Dis 2011;70:1581-6.

2. Pereira D, Peleteiro B, Araújo J, Branco J, Santos RA, Ramos E. The effect of osteoarthritis definition on prevalence and incidence estimates: a systematic review. Osteoarthritis Cartilage 2011;19:1270-85.

3. Yeşil H, Hepgüler S, Öztürk C, Çapacı K, Yeşil M. Prevalence of symptomatic knee, hand and hip osteoarthritis among individuals 40 years or older: a study conducted in İzmir city. Acta Orthop Traumatol Turc 2013;47:231-5.
4. Oppong R, Jowett S, Nicholls E, Whitehurst DG, Hill S, Hammond A, et al. Joint protection and hand exercises for hand osteoarthritis: an economic evaluation comparing methods for the analysis of factorial trials. Rheumatology (Oxford) 2015;54:876-83.

5. Becker SJ, Teunis T, Blauth J, Kortlever JT, Dyer GS, Ring D. Medical services and associated costs vary widely among surgeons treating patients with hand osteoarthritis. Clin Orthop Relat Res 2015;473:1111-7.

6. Damman W, Liu R, Kroon FPB, Reijnierse M, Huizinga TWJ, Rosendaal FR, et al. Do Comorbidities Play a Role in Hand Osteoarthritis Disease Burden? Data from the Hand Osteoarthritis in Secondary Care Cohort. J Rheumatol 2017;44:1659-66.

7. Zhang W, Doherty M, Leeb BF, Alekseeva L, Arden NK, Bijlsma JW, et al. EULAR evidence based recommendations for the management of hand osteoarthritis: report of a Task Force of the EULAR Standing Committee for International Clinical Studies Including Therapeutics (ESCISIT). Ann Rheum Dis 2007;66:377-88.

8. Kjeken I. Occupational therapy-based and evidencesupported recommendations for assessment and exercises in hand osteoarthritis. Scand J Occup Ther 2011;18:265-81.

9. Hochberg MC, Altman RD, April KT, Benkhalti M, Guyatt G, McGowan J, et al. American College of Rheumatology 2012 recommendations for the use of nonpharmacologic and pharmacologic therapies in osteoarthritis of the hand, hip, and knee. Arthritis Care Res 2012;64:465-74.

10. Manara M, Bortoluzzi A, Favero M, Prevete I, Sciré CA, Bagnato G, et al. Italian Society for Rheumatology recommendations for the management of hand osteoarthritis. Reumatismo 2013;65:167-85.

11. National Institute for Health and Clinical Excellence. Osteoarthritis: National Clinical Guideline for Care and Management in Adults. London, England: NICE; 2014. Available from: https://www.nice.org.uk/ guidance/cg177

12. Kloppenburg M, Kroon FP, Blanco FJ, Doherty M, Dziedzic KS, Greibrokk E, et al. 2018 update of the EULAR recommendations for the management of hand osteoarthritis. Ann Rheum Dis 2019;78:16-24.

13. Stoffer-Marx MA, Klinger M, Luschin S, MeriauxKratochvila S, Zettel-Tomenendal M, Nell-Duxneuner $\mathrm{V}$, et al. Functional consultation and exercises improve grip strength in osteoarthritis of the hand - a randomised controlled trial. Arthritis Res Ther 2018;20:253.

14. Kang TW, Lee JH, Park DH, Cynn HS. Effects of a finger exercise program on hand function in automobile workers with hand osteoarthritis: A randomized controlled trial. Hand Surg Rehabil 2019;38:59-66.

15. Wouters RM, Tsehaie J, Slijper HP, Hovius SER, Feitz R; Hand-Wrist Study Group, Selles RW. Exercise Therapy in Addition to an Orthosis Reduces Pain 
More Than an Orthosis Alone in Patients With Thumb Base Osteoarthritis: A Propensity Score Matching Study. Arch Phys Med Rehabil 2019;100:1050-60.

16. Malahias MA, Roumeliotis L, Nikolaou VS, Chronopoulos E, Sourlas I, Babis GC. Platelet-Rich Plasma versus Corticosteroid Intra-Articular Injections for the Treatment of Trapeziometacarpal Arthritis: A Prospective Randomized Controlled Clinical Trial. Cartilage 2018:1947603518805230.

17. Kloppenburg M, Ramonda R, Bobacz K, Kwok WY, Elewaut D, Huizinga TWJ, et al. Etanercept in patients with inflammatory hand osteoarthritis (EHOA): a multicentre, randomised, double-blind, placebocontrolled trial. Ann Rheum Dis 2018;77:1757-64.

18. Kloppenburg M, Peterfy C, Haugen IK, Kroon F, Chen S, Wang L, et al. Phase Ila, placebocontrolled, randomised study of lutikizumab, an antiinterleukin- $1 \alpha$ and anti-interleukin-1 $\beta$ dual variable domain immunoglobulin, in patients with erosive hand osteoarthritis. Ann Rheum Dis 2019;78:413-20.

19. Aitken D, Laslett LL, Pan F, Haugen IK, Otahal P, Bellamy $\mathrm{N}$, et al. A randomised double-blind placebocontrolled crossover trial of HUMira (adalimumab) for erosive hand OsteoaRthritis - the HUMOR trial. Osteoarthritis Cartilage 2018;26:880-7.

20. Cebrian-Gomez R, Lizaur-Utrilla A, SebastiaForcada E, Lopez-Prats FA. Outcomes of cementless joint prosthesis versus tendon interposition for trapeziometacarpal osteoarthritis: a prospective study. J Hand Surg Eur Vol 2019;44:151-8.

21. Buhler M, Chapple CM, Stebbings S, Sangelaji B, Baxter GD. Effectiveness of splinting for pain and function in people with thumb carpometacarpal osteoarthritis: a systematic review with meta-analysis. Osteoarthritis Cartilage 2019;27:547-59.

22. Smeraglia F, Mariconda M, Balato G, Di Donato SL, Criscuolo G, Maffulli N. Dubious space for Artelon joint resurfacing for basal thumb (trapeziometacarpal joint) osteoarthritis. A systematic review. Br Med Bull 2018; 126:79-84

23. Woolf SH, Grol R, Hutchinson A, Eccles M, Grimshaw J. Clinical guidelines: potential benefits, limitations, and harms of clinical guidelines. BMJ 1999;318:527-30.

24. Kroon FPB, Carmona L, Schoones JW, Kloppenburg M. Efficacy and safety of non-pharmacological, pharmacological and surgical treatment for hand osteoarthritis: a systematic literature review informing the 2018 update of the EULAR recommendations for the management of hand osteoarthritis. RMD Open 2018;4:e000734.

25. AGREE II. Available from: http://www.agreetrust. org/wp-content/uploads/2013/10/AGREE-IIUsers-Manual-and-23-item-Instrument_2009_ UPDATE_2013.pdf

26. Zhang Y, Niu J, Kelly-Hayes M, Chaisson CE, Aliabadi P, Felson DT. Prevalence of symptomatic hand osteoarthritis and its impact on functional status among the elderly: The Framingham Study. Am J Epidemiol 2002;156:1021-7.

27. Qin J, Barbour KE, Murphy LB, Nelson AE, Schwartz TA, Helmick CG, et al. Lifetime Risk of Symptomatic Hand Osteoarthritis: The Johnston County Osteoarthritis Project. Arthritis Rheumatol 2017;69:1204-12.

28. Michon M, Maheu E, Berenbaum F. Assessing healthrelated quality of life in hand osteoarthritis: a literature review. Ann Rheum Dis 2011;70:921-8.

29. Kwok WY, Vliet Vlieland TP, Rosendaal FR, Huizinga TW, Kloppenburg M. Limitations in daily activities are the major determinant of reduced health-related quality of life in patients with hand osteoarthritis. Ann Rheum Dis 2011;70:334-6.

30. Kroon FP, van der Burg LR, Buchbinder R, Osborne $\mathrm{RH}$, Johnston RV, Pitt V. Self-management education programmes for osteoarthritis. Cochrane Database Syst Rev 2014;(1):CD008963.

31. Dziedzic K, Nicholls E, Hill S, Hammond A, Handy J, Thomas E, et al. Self-management approaches for osteoarthritis in the hand: a $2 \times 2$ factorial randomised trial. Ann Rheum Dis 2015;74:108-18.

32. Wilder FV, Barrett JP, Farina EJ. Joint-specific prevalence of osteoarthritis of the hand. Osteoarthritis Cartilage 2006;14:953-7.

33. Marshall M, van der Windt D, Nicholls E, Myers H, Dziedzic K. Radiographic thumb osteoarthritis: frequency, patterns and associations with pain and clinical assessment findings in a community-dwelling population. Rheumatology (Oxford) 2011;50:735-9.

34. Shay LA, Lafata JE. Where is the evidence? A systematic review of shared decision making and patient outcomes. Med Decis Making 2015;35:114-31.

35. Légaré F, Witteman HO. Shared decision making: examining key elements and barriers to adoption into routine clinical practice. Health Aff (Millwood) 2013;32:276-84.

36. Brosseau L, Thevenot O, MacKiddie O, Taki J, Wells GA, Guitard P, et al. The Ottawa Panel guidelines on programmes involving therapeutic exercise for the management of hand osteoarthritis. Clin Rehabil 2018;32:1449-71.

37. Dilek B, Gözüm M, Şahin E, Baydar M, Ergör G, El $\mathrm{O}$, et al. Efficacy of paraffin bath therapy in hand osteoarthritis: a single-blinded randomized controlled trial. Arch Phys Med Rehabil 2013;94:642-9.

38. Østerås N, Kjeken I, Smedslund G, Moe RH, Slatkowsky-Christensen B, Uhlig T, et al. Exercise for Hand Osteoarthritis: A Cochrane Systematic Review. J Rheumatol 2017;44:1850-8.

39. Zeng C, Wei J, Persson MSM, Sarmanova A, Doherty M, Xie D, et al. Relative efficacy and safety of topical non-steroidal anti-inflammatory drugs for osteoarthritis: a systematic review and network meta-analysis of randomised controlled trials and observational studies. Br J Sports Med 2018;52:642-50. 
40. Verbruggen G, Goemaere S, Veys EM. Systems to assess the progression of finger joint osteoarthritis and the effects of disease modifying osteoarthritis drugs. Clin Rheumatol 2002;21:231-43.

41. Gabay C, Medinger-Sadowski C, Gascon D, Kolo F, Finckh A. Symptomatic effects of chondroitin 4 and chondroitin 6 sulfate on hand osteoarthritis: a randomized, double-blind, placebo-controlled clinical trial at a single center. Arthritis Rheum 2011;63:3383-91.

42. Bahadir C, Onal B, Dayan VY, Gürer N. Comparison of therapeutic effects of sodium hyaluronate and corticosteroid injections on trapeziometacarpal joint osteoarthritis. Clin Rheumatol 2009;28:529-33.

43. Figen Ayhan F, Ustün N. The evaluation of efficacy and tolerability of Hylan G-F 20 in bilateral thumb base osteoarthritis: 6 months follow-up. Clin Rheumatol 2009;28:535-41.

44. Kingsbury SR, Tharmanathan P, Keding A, Ronaldson SJ, Grainger A, Wakefield RJ, et al. Hydroxychloroquine Effectiveness in Reducing Symptoms of Hand Osteoarthritis: A Randomized Trial. Ann Intern Med 2018;168:385-95.

45. Kingsbury SR, Tharmanathan P, Arden NK, Batley $\mathrm{M}$, Birrell F, Cocks K, et al. Pain reduction with oral methotrexate in knee osteoarthritis, a pragmatic phase iii trial of treatment effectiveness (PROMOTE): study protocol for a randomized controlled trial. Trials 2015;16:77.

46. Rech J, Faustini F, Hueber AJ, Ochs W, Spieler W, Kellner $\mathrm{H}$, et al. A PHASE 2, Multicenter, Randomized, Double-Blind, Placebo-Controlled, Parallel-Group, Efficacy Study of Apremilast (CC10004) in Subjects with Erosive Hand Osteoarthritis. ACR/ARHP Annual Meeting November 14-19, 2014; Boston: Abstract Number: 2233.

47. Wouters RM, Tsehaie J, Hovius SER, Dilek B, Selles RW. Postoperative rehabilitation following thumb base surgery: A systematic review of the literature. Arch Phys Med Rehabil 2018;99:1177-212.e2.

48. Haugen IK, Mathiessen A, Slatkowsky-Christensen $\mathrm{B}$, Magnusson $\mathrm{K}$, Bøyesen $\mathrm{P}$, Sesseng $\mathrm{S}$, et al. Synovitis and radiographic progression in non-erosive and erosive hand osteoarthritis: is erosive hand osteoarthritis a separate inflammatory phenotype? Osteoarthritis Cartilage 2016;24:647-54.

49. Ahern M, Skyllas J, Wajon A, Hush J. The effectiveness of physical therapies for patients with base of thumb osteoarthritis: Systematic review and meta-analysis. Musculoskelet Sci Pract 2018:46-54.

50. Rillo O, Riera H, Acosta C, Liendo V, Bolaños J, Monterola L, etal. PANLAR consensus recommendations for the management in osteoarthritis of hand, hip, and knee. J Clin Rheumatol 2016;22:345-54. 